\title{
过渡金属促进的多组分串联反应用于合成复杂碳硼烷衍生物的研究进展
}

\author{
张慧芳 $a, b$ 邱早早 $*, b, c$ 谢作伟 $*, b, d$ \\ ( ${ }^{a}$ 河南师范大学化学化工学院 河南新乡 453007) \\ ( ${ }^{b}$ 中国科学院上海有机化学研究所 沪港化学合成联合实验室 上海 200032) \\ ( ${ }^{c}$ 中国科学院上海有机化学研究所 中国科学院能量调控重点实验室 上海 200032) \\ ( ${ }^{d}$ 香港中文大学化学系 香港)
}

\begin{abstract}
摘要 十二顶点碳硼烷是一类含有碳、氢及硼原子的簇合物，具有特殊的热稳定性和化学稳定性，在医药、材料、能 源、配位化学及金属有机化学中都得到广泛的应用. 因此，其官能团化成为该领域的研究热点. 其中过渡金属介导/催 化的多组分串联反应不仅具有高原子经济性的特点，还可以通过简单的原料和简便的操作，不经中间产物分离，以高 选择性直接获取复杂产物. 总结了近年来过渡金属参与的十二顶点碳硼烷 C,C-、C,B-或 B,B-顶点通过多组分串联反应 高效官能团化的研究进展, 同时对部分反应机理进行了讨论, 并就该研究领域所面临的挑战和发展前景进行了展望.
\end{abstract} 关键词 硼烷簇合物; 碳硼烷; 过渡金属; 官能团化; 串联反应

\section{Recent Advances in Transition Metal-Promoted Multicomponent Cascade Reactions for Controlled Synthesis of Complex Carborane Derivatives}

\author{
Zhang, Huifang ${ }^{a, b} \quad$ Qiu, Zaozao ${ }^{*, b, c} \quad$ Xie, Zuowei*,b,d \\ ( ${ }^{a}$ School of Chemistry and Chemical Engineering, Henan Normal University, Xinxiang, Henan 453007) \\ ( ${ }^{b}$ Shanghai-Hong Kong Joint Laboratory in Chemical Synthesis, Shanghai Institute of Organic Chemistry, \\ Chinese Academy of Sciences, Shanghai 200032) \\ ( ${ }^{c}$ CAS Key Laboratory of Energy Regulation Materials, Shanghai Institute of Organic Chemistry, \\ Chinese Academy of Sciences, Shanghai 200032) \\ ( ${ }^{d}$ Department of Chemistry, The Chinese University of Hong Kong, Hong Kong)
}

\begin{abstract}
Carboranes are a class of carbon-boron molecular clusters with exceptional thermal and chemical stabilities. They are finding a variety of applications in medicine, materials, and coordination/organometallic chemistry as functional building blocks. To this end, the selective functionalization of carboranes has received growing research interests. Transition metal-promoted multicomponent cascade reactions are particularly attractive since they have the advantages of step- and atomeconomy for the construction of complex products from readily available starting materials by simple operations. The recent advances in transition metal-promoted multicomponent cascade reactions for selective functionalization of carboranes are summarized in this focus review. The related reaction mechanisms and challenges in this research area are also discussed.

Keywords borane cluster; carborane; transition metal; functionalization; cascade reaction
\end{abstract}

二十面体碳硼烷是一类具有 26 个离域电子的硼碳 簇合物, 它们与苯有许多相似的性质, 例如疏水性、芳 香性、优异的热稳定性和化学稳定性. 另一方面, 碳硼
烷有其独有的性质，例如几何球面、三维电子离域等 ${ }^{[1]}$. 这些特质使碳硼烷及其衍生物得到了广泛的应用，例 如：碳嗍烷化合物在治疗癌症的嗍中子俘获疗法

* Corresponding authors. E-mail: qiuzz@sioc.ac.cn; zxie@cuhk.edu.hk

Received May 28, 2020; revised June 15, 2020; published online June 19, 2020.

Dedicated to Professor Henry N. C. Wong on the occasion of his 70th birthday.

Project supported by the National Natural Science Foundation of China (No. 21772223), the Science and Technology Commission of Shanghai Municipality (No. 18590760800), the Chinese Academy of Sciences, and the Hong Kong Research Grants Council (No. 14306519).

国家自然科学基金(No. 21772223)、上海市科学技术委员会(No. 18590760800)、中国科学院、香港研究资助局(No. 14306519)资助项目. 
$(\mathrm{BNCT})$ 中用作高硼含量药物 ${ }^{[2]}$; 在金属有机化学中用 作多变配体 ${ }^{[3]}$; 在超分子及纳米材料中用作功能砌块 ${ }^{[4]}$; 在有机光学材料中用作电子阱等 ${ }^{[5]}$. 为了拓展其应用, 高效获取碳硼烷官能化衍生物已成为碳嗍烷化学领域 的重要研究方向.

在碳顶点官能团化方面, 十硣烷与取代炔烃反应可 以直接制备取代邻一碳硼烷 ${ }^{[6]}$; 另一方面, 由于碳硼烷上 $\mathrm{CH}$ 具有一定的酸性, 容易被强碱拔除质子, 生成的碳 嗍烷阴离子与亲电试剂进一步反应, 是制备多种碳顶点 取代碳嗍烷衍生物的有效方法 ${ }^{[7]}$. 近年来, 过渡金属介 导或催化的碳顶点官能团化方法得到了迅速的发展, 为 碳-取代碳硼烷衍生物的合成提供了有效的方法 ${ }^{[8]}$.

而相对于已发展较为成熟的碳顶点官能团化, 碳喼 烷硼顶点活化及进一步衍生化的研究较为滞后, 目前 嗍一取代碳嗍烷化合物的种类及数量都较为有限, 大大 限制了后续的性质研究和应用开发. 近年来, 除了直接 对硼顶点进行亲核取代反应外 ${ }^{[9]}$, 碳硼烷 $\mathrm{B}-\mathrm{H}$ 键催化 官能团化过程也受到了合成化学家们的广泛关注, 相继 报道了过渡金属催化的系列区域选择性 $\mathrm{B}-\mathrm{H}$ 键芳基 化 ${ }^{[10]}$ 、烯基化 ${ }^{[11]}$ 、炔基化 ${ }^{[12]}$ 、嗍基化 ${ }^{[13]}$ 、氟化 ${ }^{[14]}$ 、氧

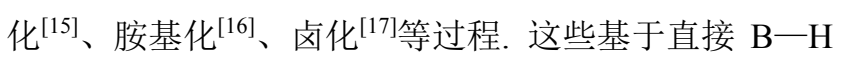
键活化的合成策略可以简化原料, 缩短反应流程, 从而 提升合成效率和原子经济性 ${ }^{[18]}$.

多组分反应、串联反应及一锅法反应的系列合成方 法都具有高原子及步骤经济性，原料简单易得，操作简 便等特点, 可以不经中间产物分离, 直接得到结构复杂 的最终产物. 在以上一系列过渡金属参与的碳硼烷区域 选择性官能团化方法学报道中, 除了可以引入单一种类 的取代基外，也能通过多组分反应、串联反应及一锅法 反应的策略, 以双取代、多取代等不同形式实现复杂碳 嗍烷衍生物的高效构筑. 本文将围绕 C, C, C,B, B,B三种 不同取代类型，综述过渡金属参与的复杂碳硼烷衍生物 可控合成的研究进展, 并对该领域所面临的挑战及发展 前景做出了总结和展望.

\section{1 碳碳一取代碳硼烷衍生物的合成}

碳硼炔(1,2-脱氢碳硼烷)可以看作苯炔的三维类似 物, 而过渡金属一邻一碳喼炔配合物中金属一碳键具有不 同于经典金属一碳键的化学性质, 能与多种不饱和分子 发生反应，其反应模式取决于中心金属离子的电子构 型 ${ }^{[19]} .2009$ 年, 谢作伟课题组 ${ }^{[20]}$ 开发了镍介导的碳硼炔 与烯烃和炔烃的三组分 $[2+2+2]$ 环加成反应. 在该反 应体系中, 邻碳嗍烷在丁基锂的作用下得到碳硼烷二锂 盐, 其与 $\mathrm{NiCl}_{2}\left(\mathrm{PPh}_{3}\right)_{2}$ 通过盐消除原位制得镍一碳硼炔配 合物 1.1 同时与过量的烯烃和炔烃在加热条件下可以得
到一系列二氢苯并碳硼烷衍生物 2 (Scheme 1). 其中, 参与反应的烯烃可以是 2-乙烯基吡啶或丙烯酸甲酯 $\left(\mathrm{R}^{1}=2-\mathrm{Py}, \mathrm{CO}_{2} \mathrm{Me}\right)$, 而炔烃则需是烷基或芳基取代的中 间炔 $\left(\mathrm{R}^{2}, \mathrm{R}^{3}=\right.$ alkyl, aryl), 产物分离收率最高可达 $59 \%$. 该反应的路径首先是烯烃插入镍一碳硼炔 $\mathbf{1}$ 的 $\mathrm{Ni}-\mathrm{C}$ 键, 得到碳硼烷并镍杂环戊烷中间体 $\mathbf{A}$ ，随后炔烃插入 $\mathrm{Ni}-\mathrm{C}$ (烷基)键，形成镍杂七元中间体 $\mathbf{B}$, 最后经还原消 除得到最终产物 2. 反应中由于活化烯烃的反应活性高 于炔烃，使得三组分反应可以获得优秀的化学选择性; 而由于烯烃取代基团的配位作用和烷基-芳基取代的非 对称炔烃具有一定的极性, 它们的插入步骤也分别具有 优秀的区域选择性. 由于活化烯烃中配位基团与金属中 心的作用，稳定反应中间体 $\mathbf{A}$ 可以由 $\mathbf{1}$ 与烯烃的单独反 应制备，其中 2-乙烯基吡啶的产物 A-1 的结构经单晶 X 射线衍射确证. A 与炔烃的反应也能以大于 $90 \%$ 的收率 获得最终产物，进一步证明了三组分反应获得二氢苯并 碳硼烷 2 的路径.

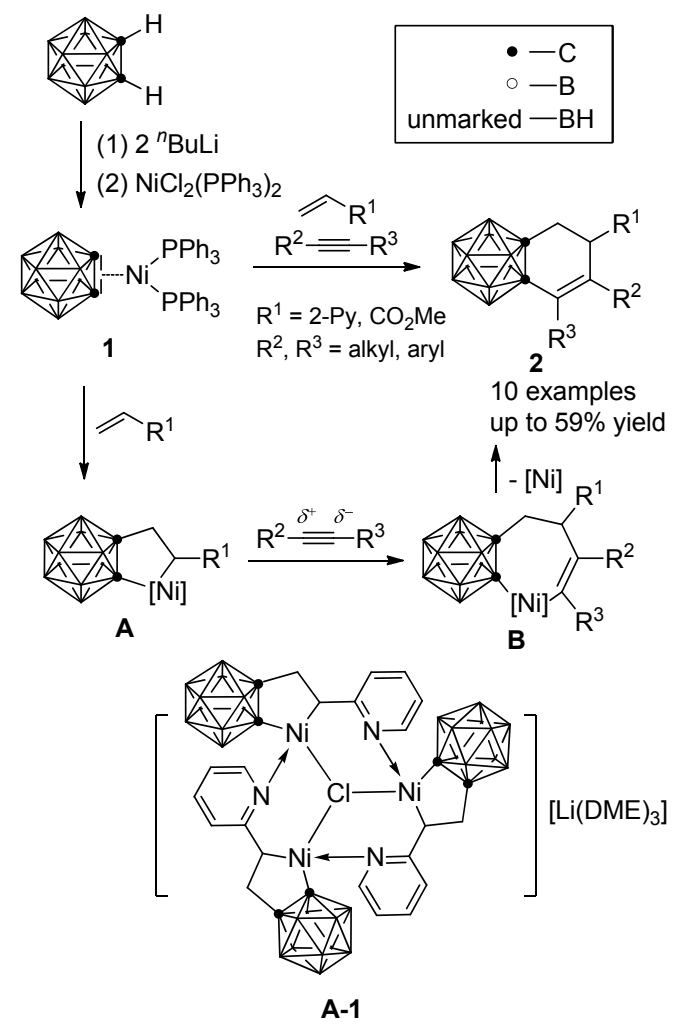

图式 1 镍一介导碳硼炔、烯烃、炔烃的三组分环加成反应 Scheme 1 Nickel-mediated three-component cycloaddition reaction of carboryne, alkenes and alkynes

过渡金属一碳硼炔配合物的反应性质取决于金属中 心的性质, 其中镍-碳硼炔 1 与炔烃作用可以获得与两

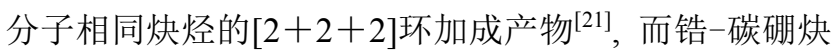
前体 3 只能与一分子炔烃发生 $\mathrm{Zr}-\mathrm{C}$ 键插入反应，得到 稳定的碳硼烷并锆杂环戊烯产物 $4^{[22]}$. 此锆杂五元环化 
合物并不能进一步与炔烃反应, 考虑到镍杂五元环化合 物更高的反应活性, 4 在 $\mathrm{NiCl}_{2}\left(\mathrm{PMe}_{3}\right)_{2}$ 存在下可以与另一 分子炔烃反应(Scheme 2) ${ }^{[23]}$ : 通过转金属作用先得到镍 杂环戊烯中间体 $\mathbf{C}$, 炔烃插入 $\mathrm{Ni}-\mathrm{C}$ (烯基)键形成镍杂 七元中间体 $\mathbf{D}$, 再经还原消除, 最终得到过渡金属锆与 镍共同介导下碳硼炔与两分子不同炔烃 $[2+2+2]$ 环加 成产物 5. 该反应同样具有优秀的炔烃插入区域选择性, 可以以最高 $85 \%$ 的分离收率得到不同取代的苯并碳硼 烷衍生物. 而通过双齿配体 dppe 的稳定作用, 可以分离 到镍转金属中间体 C-1, 其结构经由单晶 X 射线衍射确 证(Scheme 3).
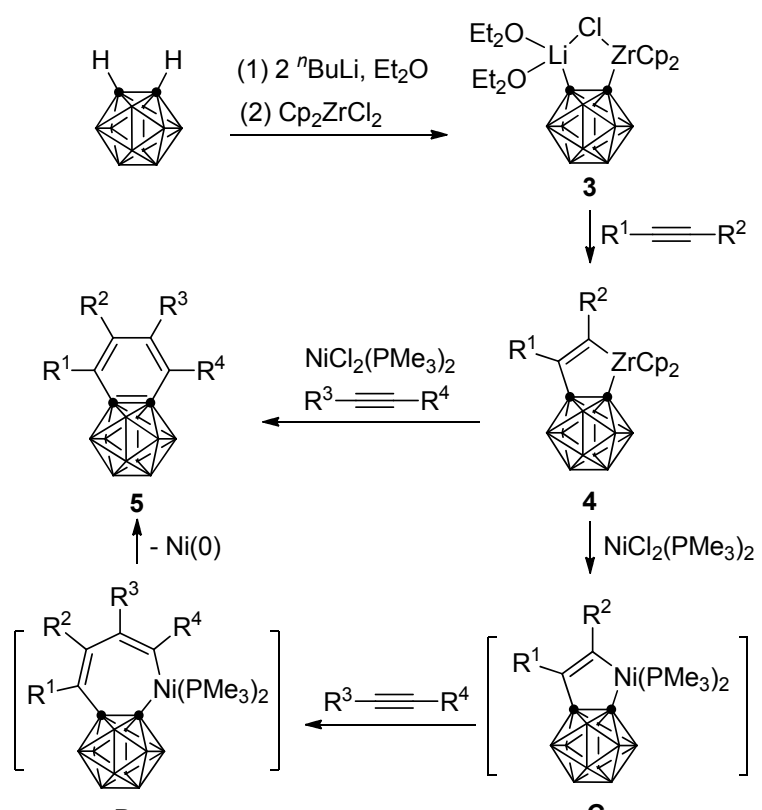

D

$\mathrm{R}^{1}, \mathrm{R}^{2}, \mathrm{R}^{3}, \mathrm{R}^{4}=$ alkyl, aryl

21 examples, up to $85 \%$ yield

图式 2 锆、镍共同介导的碳硼炔与炔烃的 $[2+2+2]$ 环加成反 应

Scheme 2 Zirconium/nickel-co-mediated $[2+2+2]$ cycloaddition reaction of carboryne and alkynes
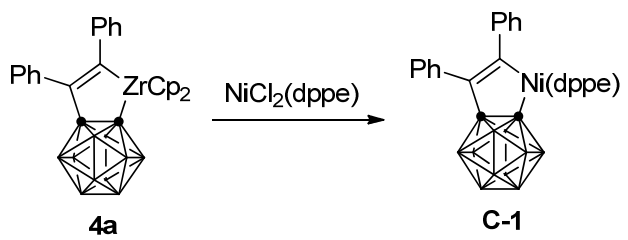

图式 3 转金属反应中间体 C-1 的合成

Scheme 3 Synthesis of transmetallation intermediate C-1

2009 年, 谢作伟课题组 ${ }^{[24]}$ 在先前工作基础上进一 步研究了锆环戌烯 $\mathbf{4 b}$ 的反应性质(Scheme 4). 一方面, 将 4b 与 2,6-二甲苯基异腈(XylNC)在甲苯中回流, 异腈 碳很容易地插入到 $\mathrm{Zr}-\mathrm{C}$ (烯基)键中, 以 $77 \%$ 的产率得 到碳硼烷并五元环产物 6. 另一方面, 在 $\mathrm{CuCl}$ 存在下,
锆杂环戌烯 $4 b$ 与邻二碘苯共同加热得到䒺并碳硼烷产 物 7, 产率为 $81 \%$, 其结构经单晶 X 射线衍射证实. 这个 反应过程中铜是必须的, 若没有 $\mathrm{CuCl}$ 的存在, 则反应 无法进行.

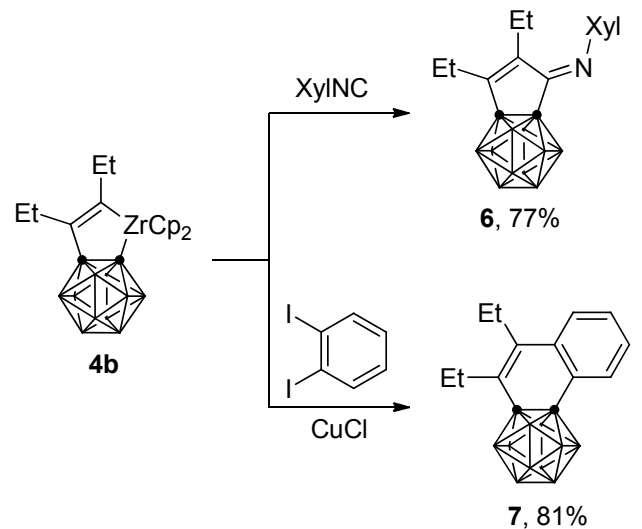

图式 4 碳硼烷并锆杂环戊烯中间体与异腈及邻二碘苯的反 应

Scheme 4 Reactions of carboranozirconacyclopentene intermediate with isocyanide and $o$-diiodobenzene

与炔烃的反应类似，锆-碳硼炔前体 $\mathbf{3}$ 同样能与一 分子末端烯烃发生 $\mathrm{Zr}-\mathrm{C}$ 键插入反应，得到稳定的碳硼 烷并锆杂环戊烷 $\mathbf{8}^{[25]}$, 插入反应的区域选择性由烯烃上 取代基的电性决定(Scheme 5): 给电子烷基位于金属 $\beta$ 位 $(\mathbf{8 a})$ 而吸电子芳基位于金属 $\alpha$ 位 $(\mathbf{8 b})$. 同样 $\mathbf{8}$ 与炔烃在 $\mathrm{NiCl}_{2}$ 存在下进一步反应, 能以最高 $88 \%$ 收率合成二氢 苯并碳硼烷 $\mathbf{9}^{[26]}$. 该方法既高效又实用, 可以从锆-碳硼 炔前体 3 出发, 与烯烃和炔烃一锅法合成二氢苯并碳嗍 烷衍生物 9a 和 9b. 值得注意的是, 在没有炔烃的情况 下, 8 与 $\mathrm{NiCl}_{2}$ 直接作用生成含有镍杂环戊烷中间体 $\beta-\mathrm{H}$ 消除产物在内的复杂混合物. 而锆杂环戊烷的镍转金属 中间体 10 同样可经由 dppe 配体稳定并得到分离鉴定, 它可以在炔烃的作用下高效转化为 $9 a$ (Scheme 6), 这也 进一步证明了此一锅法反应的路径.

当上述反应中使用芳基和三甲硅基取代炔烃时，在 $\mathrm{NiCl}_{2}\left(\mathrm{PMe}_{3}\right)_{2}$ 存在下可以最高可达 $86 \%$ 的收率获得系列 二氢富烯并碳嗍烷化合物 11 (Scheme 7) ${ }^{[27]}$. 在对该特殊 $[2+2+1]$ 环加成反应的机理研究中, 林振阳小组和谢 作伟小组 ${ }^{[28]}$ 共同通过密度泛函理论(DFT)对过渡态能量 进行了计算. 结果表明, 亚乙烯基镍过渡态在能量上较 为不利, 反应历经炔烃插入、 $\eta^{1}$-烯基至 $\eta^{2}$-烯基重排、硅 基迁移的系列过程，而炔烃上芳基取代基的位阻效应决 定最终 $[2+2+1]$ 环加成产物中环外双键的顺反构型.

2018 年, 谢作伟、邱早早课题组 ${ }^{[29]}$ 又进一步探索了 碳硼烷并锆环戊烷 8a 对一系列底物的反应性(Scheme 8). 例如, 8a 与过量硫族元素单质在 $110{ }^{\circ} \mathrm{C}$ 甲苯中反应 
9a

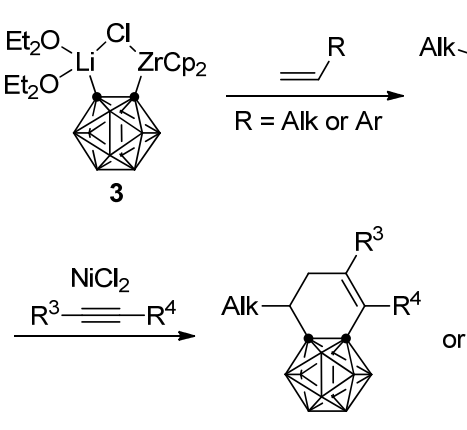

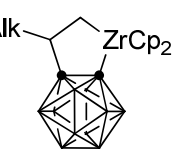

$8 a$

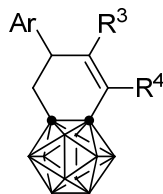

9b
$\mathrm{R}^{3}, \mathrm{R}^{4}=$ alkyl, aryl

24 examples, up to $88 \%$ yield

图式 5 锆、镍共同介导的碳硼炔与烯烃、炔烃的一锅法 $[2+$ $2+2$ ]环加成反应

Scheme 5 Zirconium/nickel-co-mediated one-pot $[2+2+2]$ cycloaddition reaction of carboryne, alkenes and alkynes

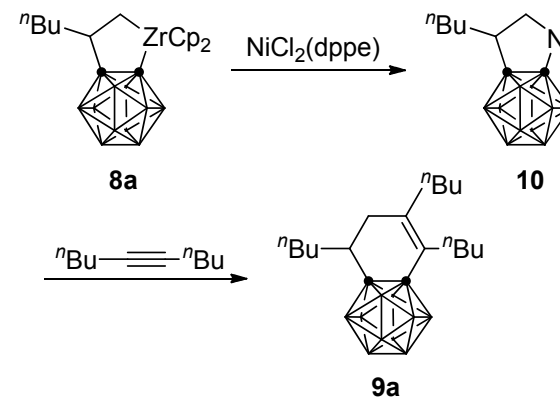

图式 6 转金属反应中间体 $\mathbf{1 0}$ 的合成

Scheme 6 Synthesis of transmetallation intermediate $\mathbf{1 0}$

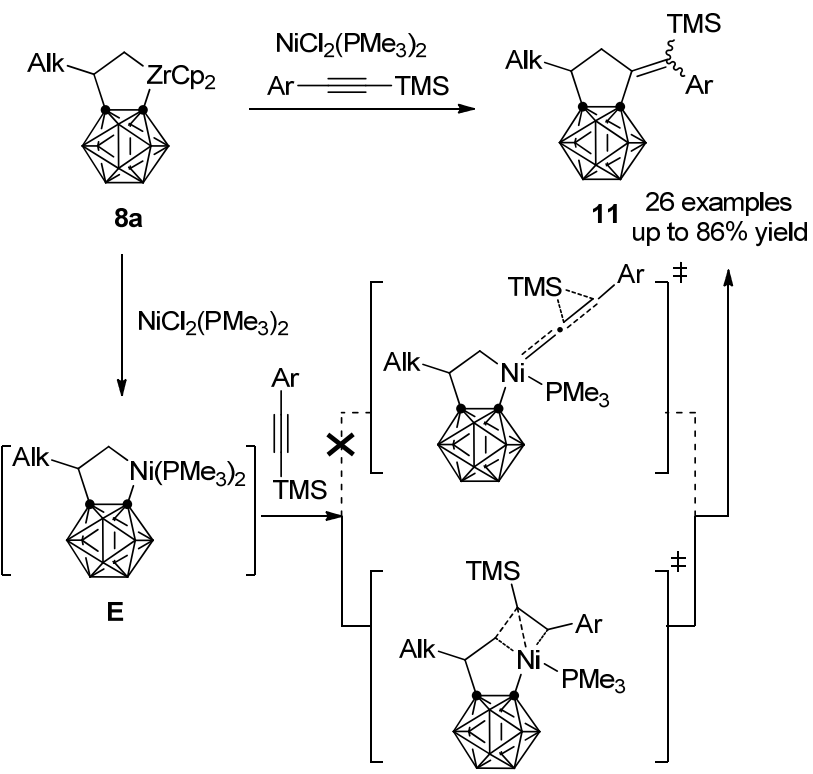

图式 7 锆、镍共同介导的碳硼炔与烯烃、三甲硅基炔烃的一 锅法 $[2+2+1]$ 环加成反应

Scheme 7 Zirconium/nickel-co-mediated one-pot $[2+2+1]$ cycloaddition reaction of carboryne, alkenes and trimethylsilylalkynes
得到碳硼烷并硫族元素杂环戊烷 12a 12c. 8a 与一氧化 碳在 $\mathrm{NiCl}_{2}\left(\mathrm{PPh}_{3}\right)_{2}$ 存在条件下得到插羰产物 $13 \mathrm{a}$, 而具有 较强活性的异腈 XylNC 在室温下插入 $8 \mathbf{a}$ 的 $\mathrm{Zr}-\mathrm{C}$ 键中, 形成亚胺环戊烷 14a. 另一方面, $8 \mathbf{a}$ 还可以与 $\mathrm{PhPCl}_{2}$ 、 ${ }^{n} \mathrm{Bu}_{2} \mathrm{SnCl}_{2}$ 和 $\mathrm{Me}_{2} \mathrm{GeCl}_{2}$ 在不同条件下通过转金属反应分 别以 $94 \%$ 、89\%和 76\%的收率得到 15 17. 这些实验结 果表明, 碳硼烷并锆杂环戊烷化合物与苯并锆杂环戊烷 在反应性上有相似之处，可以作为合成子用于合成系列 碳硼烷并碳环和杂环衍生物. 另一方面由于大位阻碳硼 烷基团的存在, $8 \mathbf{a}$ 也表现出一些自己独特的反应性质.

\section{2 碳硼一取代碳硼烷衍生物的合成}

2014 年, 谢作伟课题组 ${ }^{[30]}$ 在之前的锆、镍共同介导 碳嗍炔与烯烃、三甲硅基芳基乙炔的一锅法 $[2+2+1]$ 环加成反应基础上，发现当炔烃的芳基取代基邻位连有 溴取代基团时，最终得到 C,C,B-取代的碳硼烷并三环化 合物. 该反应的机理如 Scheme 9 所示, 碳硼烷并锆杂环 戊烷发生系列金属交换和炔烃插入重排过程后得到 $[2+2+1]$ 环加成中间体 $\mathbf{F}$. 该过程中生成的 $\mathrm{Ni}(0)$ 与

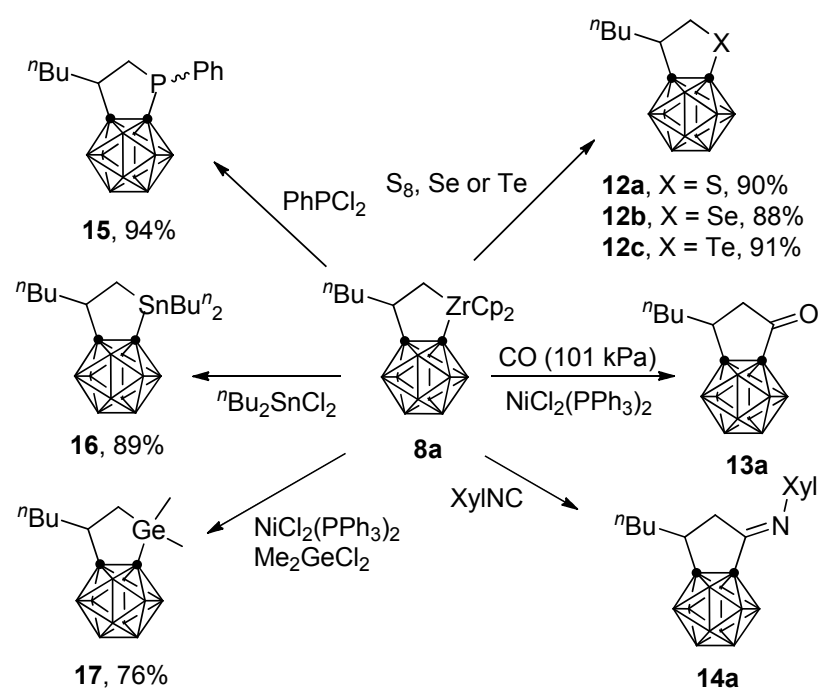

图式 8 通过碳硼烷并锆杂环戊烷对系列碳硼烷并碳环和杂 环衍生物的合成

Scheme 8 Synthesis of carborane-fused carbo- and heterocycles via zirconacyclopentane intermediate

$\mathrm{C}\left(\mathrm{sp}^{2}\right)-\mathrm{Br}$ 键发生氧化加成得到中间体 $\mathbf{G}$, 并对 $\mathrm{B}(7)-$ $H$ 或 $B(11)-H$ 进行分子内亲电取代, 在碱的作用下消 除一分子 $\mathrm{HBr}$ 得到中间体 $\mathbf{H}$, 再经还原消除生成最终产 物 18. 提前淬灭反应并不能观察到体系中 $\mathbf{F}$ 的存在, 这 表明整个串联反应过程中 $\mathrm{B}-\mathrm{C}\left(\mathrm{sp}^{2}\right)$ 偶联步骤非常高效. 这是首个通过金属催化 $\mathrm{B}-\mathrm{H}$ 键活化实现的嗍顶点芳基 化过程. 


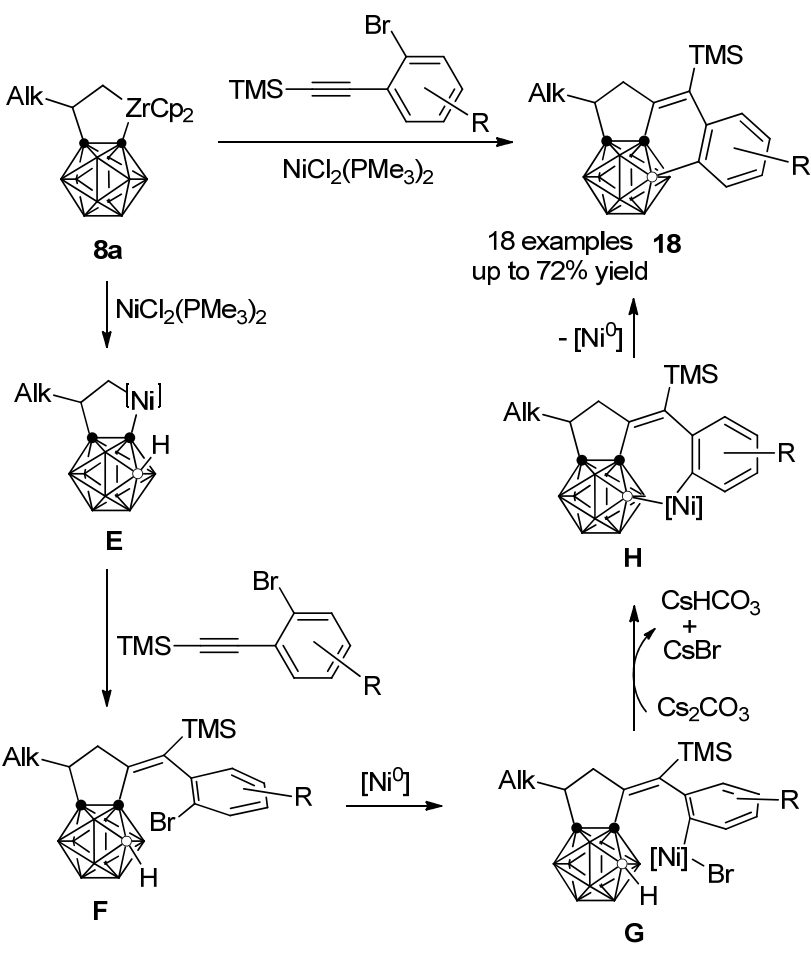

图式 9 过渡金属介导 $[2+2+1]$ 环加成与 $\mathrm{B}-\mathrm{H}$ 键芳基化串 联环化反应

Scheme 9 Transition-metal-co-mediated $[2+2+1]$ cyclization and $\mathrm{B}-\mathrm{H}$ arylation cascade cyclization

2013 年, 燕红课题组 ${ }^{[31}$ 报道了一类钴介导的 B-H 键活化制备砋官能团化碳嗍烷衍生物过程. 该反应采用 16 电子的二硫代碳硼烷钴配合物 19, 与炔烃反应形成 两种 $\mathrm{B}(3)$ 或 $\mathrm{B}(6)$ 连有降冰片烯基的 17 电子钴配合物 20 和 21 (Scheme 10). 经 DFT 理论计算证明, 反应经过过 渡金属引发的 $\mathrm{B}-\mathrm{H}$ 键活化、 $\mathrm{B}-\mathrm{Cp}$ 偶联、 $\mathrm{Cp}$ 迁移和 Diels-Alder 加成. 20 和 21 可以与第二分子炔烃进一步发 生 $\mathrm{Co}-\mathrm{S}$ 键插入反应, 最终得到金属 $\mathrm{Co}$ 消除的烯基硫 醚化合物 22 和 23 . 特别注意的是, 19 在温和条件下可以 与过量的炔烃作用, 一锅法合成复杂的 C,C,B-取代邻碳 嘲烷衍生物 22 和 23.

16 电子的二硫代碳嗍烷钴配合物 19 也能与丙炔酸 甲酯和另一 $3 \mathrm{e}^{-}$配体经三组分反应在室温下制备 B-环戊 二烯基偶联产物 24 27 (Scheme 11) ${ }^{[32]}$. 作者通过氝代 控制反应提出以下反应机理: 活化炔烃首先插入其中一 个 $\mathrm{Co}-\mathrm{S}$ 键, 并引发 $\mathrm{B}(3)-\mathrm{H}$ 键活化和对烯烃的氢转移 过程; 在 $3 \mathrm{e}^{-}$配体的作用下, $\mathrm{Cp}$ 基团的配位方式由 $\eta^{5}$ 转 变为 $\eta^{1}$, 之后再经过 $\alpha-\mathrm{H}$ 消除、 $\mathrm{H}_{2}$ 释放、 $\mathrm{Co}-\mathrm{B}$ 和 $\mathrm{Co}-\mathrm{C}$ 键断裂、 $\mathrm{B}-\mathrm{C}$ 键形成、 $\mathrm{Cp}$ 基团重新回到 $\eta^{5}$-配 位的系列过程, 得到硼顶点被 $\eta^{5}-\mathrm{Cp}$ 取代的最终产物. 本方法为复杂硼官能团化碳嗍烷衍生物的合成提供了 一条简单、高效的合成路线.

2010 年, 谢作伟课题组 ${ }^{[33]}$ 发展了钯、镍共同催化的

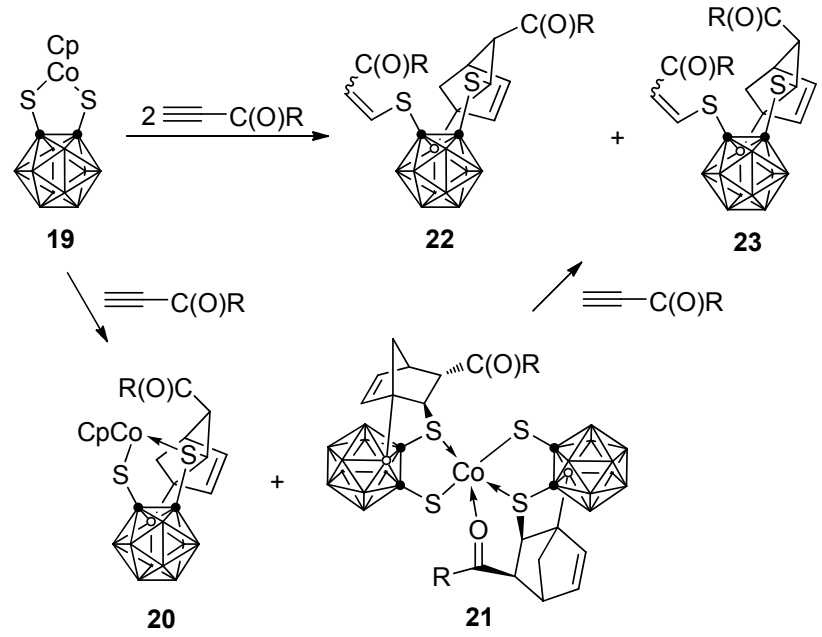

图式 10 通过 $\mathrm{B}-\mathrm{H}$ 键活化合成 B-冰片烯基取代邻-碳硼烷 Scheme 10 Synthesis of B-norbornyl $o$-carboranes via B-H activation

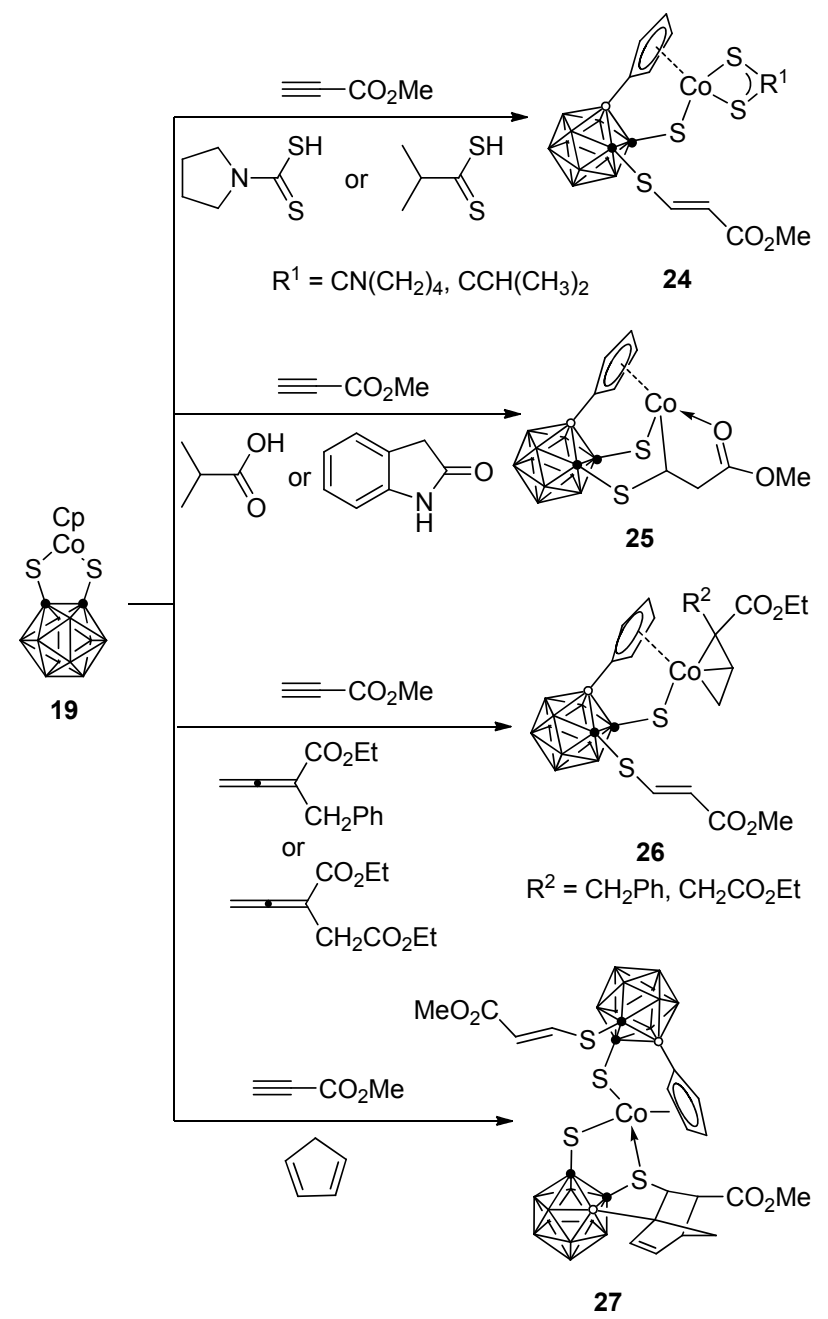

图式 11 钴介导邻一碳硼烷 $\mathrm{B}-\mathrm{H}$ 键活化与环戊二烯基 $\mathrm{C}-\mathrm{H}$ 键活化参与的三组分反应

Scheme 11 Co-mediated three-component reactions incorporating with $o$-carborane $\mathrm{B}-\mathrm{H}$ activation and cyclopentadiene $\mathrm{C}-\mathrm{H}$ activation 
1,3 -脱氢-邻-碳硼烷 (C,B-碳硼炔) 与炔烃的 $[2+2+2]$ 环 加成反应, 以最高可达 $85 \%$ 的分离收率制备 $\mathrm{C}, \mathrm{B}$-取代苯 并一邻一碳硼烷衍生物 29 和 30. 该方法为碳硼烷的碳硼 顶点同时官能团化提供了一种新的方法(Scheme 12). 一个碳顶点连有取代基的 3-碘代-邻-碳硼烷 28 在催化 剂存在下不能与炔烃发生反应, 需要与正丁基锂作用生 成 1-锂-3-碘代-邻-碳硼烷后才能发生与炔烃的 $[2+2+$ 2]环加成反应, 因而本反应是经由金属-C,B-碳嗍炔中 间体进行的. 由于 $\mathrm{Ni}(0)$ 与 $\mathrm{B}-\mathrm{I}$ 键很难发生插入反应, 因此 1-锂-3-碘代-邻-碳硼烷前体的 B - I 键与 $\operatorname{Pd}(0)$ 发生 氧化加成得到中间体 $\mathbf{I}$, 消除 LiI 形成 Pd-1,3-脱氢-邻碳嗍烷中间体 $\mathbf{J}$. 由于双组分 $\mathrm{Pd} / \mathrm{Ni}$ 催化剂体系在 1,3脱氢一邻一碳硼烷与炔的反应中比单独的 Pd 催化剂更有 效, 因此反应过程中可能存在 $\mathrm{Pd}$ 与 $\mathrm{Ni}$ 之间的转金属过 程得到 Ni-1,3-脱氢- 邻-碳硼烷中间体 $\mathbf{K}$. 由于 $M-B$ 键 较 $\mathrm{M}-\mathrm{C}$ 键亲核性更高, 炔烃首先插入 $\mathrm{M}-\mathrm{B}$ 键得到镍 杂环戊烯中间体 $\mathbf{L}$, 第二分子的炔烃以两种形式插入 $\mathrm{Ni}-\mathrm{C}$ 键形成 $\mathbf{M}\left(\mathbf{M}^{\prime}\right)$, 最后还原消除得到产物和 $\mathrm{Ni}(0)$ 完成催化循环(Scheme 13).

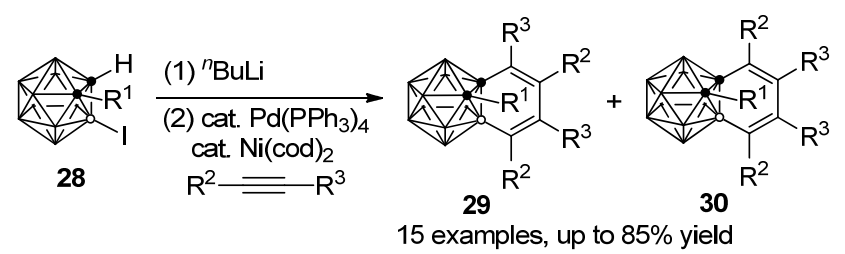

图式 12 钯、镍-共同催化的 1,3-脱氢-邻-碳硼烷与炔烃环加 成反应

Scheme $12 \mathrm{Pd} / \mathrm{Ni}$-cocatalyzed cycloaddition of 1,3-dehydro-ocarborane with alkynes

2018 年, 谢作伟课题组 ${ }^{[34]}$ 报道了铑催化的邻一碳硼 烷基- $N$-芳基亚胺 31 与乙烯基酮的环化反应，可以通过 串联反应高效地合成氮杂多环并碳硼烷的衍生物 32 (Scheme 14). 该反应采用 $\mathrm{Cu}(\mathrm{OPiv})_{2}$ 和 $\mathrm{AgSbF}_{6}$ 作为添加 剂, 同时在 $\mathrm{Mes} \mathrm{COOH}$ 的存在下, 利用 $\mathrm{Rh}(\mathrm{III})$ 邻碳硼烷 $\mathrm{B}(4)-\mathrm{H}$ 键和亚胺的 $N$-芳基 $\mathrm{C}-\mathrm{H}$ 键依次活化, 最终以 高达 $74 \%$ 的收率得到目标产物. 提前淬灭本反应可以分 离到亚胺导向的烯烃与邻碳硼烷的 $\mathrm{B}-\mathrm{H}$ 键活化-环化 中间体 33, 并经单晶 $\mathrm{X}$ 射线衍射鉴定了其结构. 证明了 碳硼烷 $\mathrm{B}-\mathrm{H}$ 键的活化优先于芳基 $\mathrm{C}-\mathrm{H}$ 键, 并通过芳 基気代底物反应的动力学同位素效应 $(\mathrm{KIE})$ 值 $\left(k_{\mathrm{H}} / k_{\mathrm{D}}=\right.$ 1.7)确认 $\mathrm{C}-\mathrm{H}$ 键活化并非本串联反应过程中的决速步. 以此为基础, 作者提出了以下可能的反应机理(Scheme 15): 底物 31 首先在亚胺导向基的作用下, $\mathrm{Rh}(\mathrm{III})$ 亲电进 攻富电子的 $\mathrm{B}(4 / 5)-\mathrm{H}$ 生成铑杂五元环中间体 $\mathbf{N}$, 烯烃 插入 $\mathrm{B}-\mathrm{Rh}$ 键得到中间体 $\mathrm{O}$, 其中 $\mathrm{C}-\mathrm{Rh}$ 和 $\mathrm{C}=\mathrm{N}$ 间 的分子内亲核环化形成 $\mathbf{P}$, 随后经由质子化得到 $\mathbf{3 3}$ 并释

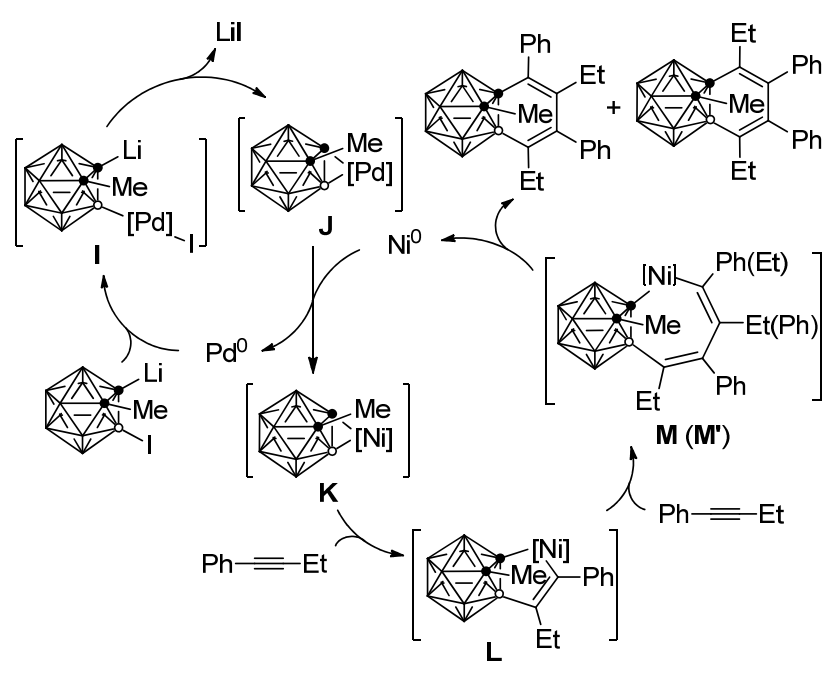

图式 13 钯、镍-共同催化 $[2+2+2]$ 环加成反应机理 Scheme 13 Proposed mechanism for $\mathrm{Pd} / \mathrm{Ni}$-cocatalyzed $[2+$ $2+2]$ cycloaddition

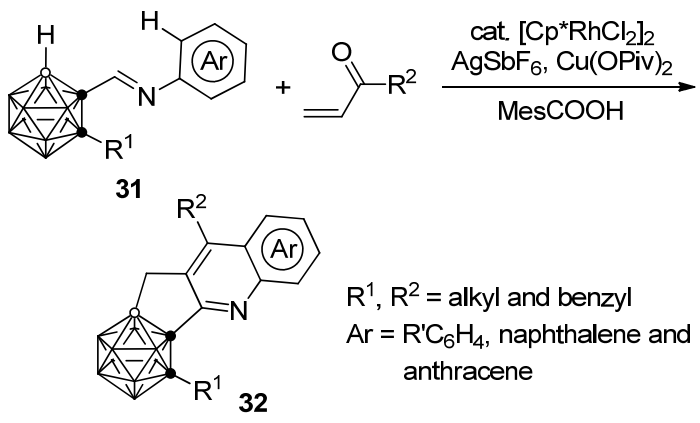

26 examples, up to $74 \%$ yield

图式 14 铑催化 $\mathrm{B}-\mathrm{H}$ 和 $\mathrm{C}-\mathrm{H}$ 键串联活化合成氮杂多环并 碳硼烷

Scheme 14 Synthesis of carborane-fused $\mathrm{N}$-polyheterocycles by rhodium catalyzed cascade $\mathrm{B}-\mathrm{H}$ and $\mathrm{C}-\mathrm{H}$ activation

放催化剂. 接下来 $\mathrm{Rh}(\mathrm{III})$ 催化芳基 $\mathrm{C}-\mathrm{H}$ 键活化形成铑 杂八元环中间体 $\mathbf{Q}$, 随后 $\mathrm{C}-\mathrm{Rh}$ 和 $\mathrm{C}=\mathrm{O}$ 的分子内亲核 环化和质子化得到 $\mathbf{S}$ 并释放 $\mathrm{Rh}$ 催化剂，最后 $\mathbf{S}$ 经脱水 及氧化芳构化得到最终产物 32. 该过程中铑催化剂的 价态始终保持不变.

\section{3 嗍嗍一取代碳硼烷衍生物的合成}

由于碳硼烷笼上十个 BH 的化学环境非常相似，因 此对两个嗍顶点进行连续可控官能团化具有更高的挑 战性. 2019 年, 谢作伟课题组 ${ }^{[35]}$ 报道了铱催化羧基碳硼 烷 34 与苯甲酰胺连续脱氢偶联反应合成碳硼烷并异喹 啉酮衍生物的方法(Scheme 16). 该反应采用 $\left[\mathrm{Cp}^{*} \mathrm{IrCl}_{2}\right]_{2}$ 为催化剂, 在 $\mathrm{AgOAc} 、 \mathrm{Li}_{2} \mathrm{CO}_{3}$ 和 $\mathrm{Cu}(\mathrm{OPiv})_{2}$ 存在下, 经 过 $\mathrm{B}-\mathrm{H} / \mathrm{C}-\mathrm{H}$ 和 $\mathrm{B}-\mathrm{H} / \mathrm{N}-\mathrm{H}$ 的两步串联脱氢偶联反 应，高效制备碳硼烷并异喹啉酮衍生物 35, 最高收率可 达 $91 \%$. 此外在适合的反应条件下，该连续脱氢偶联反 


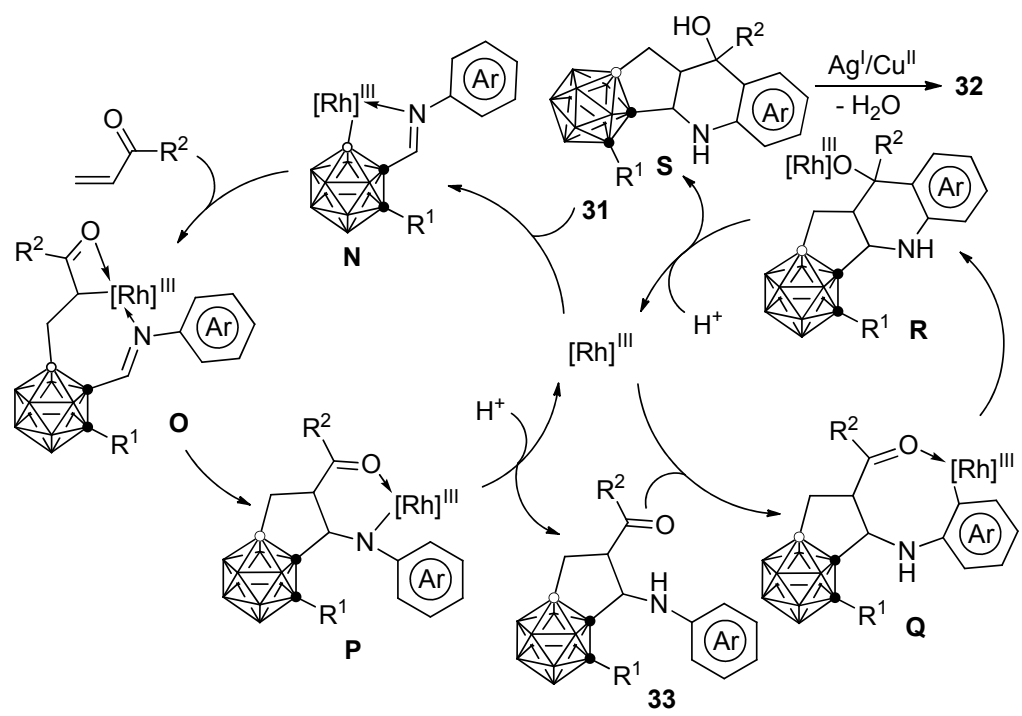

图式 15 铑催化 $\mathrm{B}-\mathrm{H}$ 和 $\mathrm{C}-\mathrm{H}$ 键串联活化环化机理

Scheme 15 Proposed mechanism for rhodium catalyzed cascade B-H/C $-\mathrm{H}$ activation and cyclization

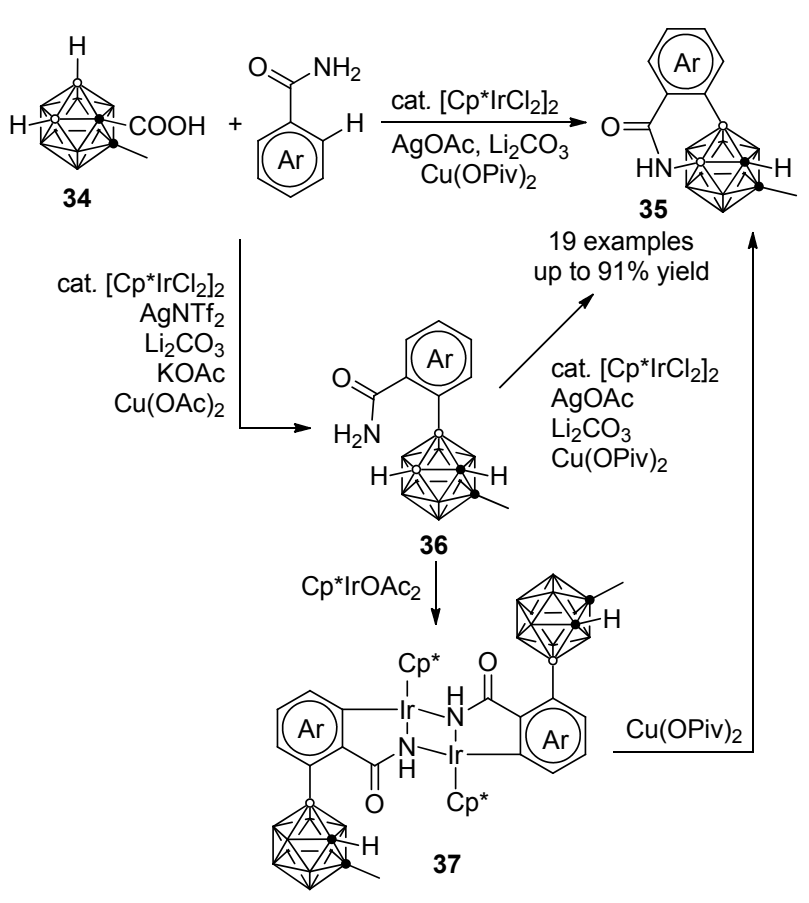

图式 16 铱催化 $\mathrm{BH} / \mathrm{CH}$ 和 $\mathrm{BH} / \mathrm{NH}$ 串联脱氢交叉偶联合成碳 硼烷并异喹啉酮

Scheme 16 Synthesis of carborano-isoquinolinones via iridium catalyzed cascade dehydrogenative cross-coupling of $\mathrm{BH} / \mathrm{CH}$ and $\mathrm{BH} / \mathrm{NH}$

应可以停留在第一个 $\mathrm{B}-\mathrm{H} / \mathrm{C}-\mathrm{H}$ 交叉偶联步骤, 得到 产物 36. 值得注意的是, 该反应中羧基导向基在 $\mathrm{B}-\mathrm{C}$ 交叉偶联步骤中起关键作用, 并随后通过原位脱羧去 除. 36 直接与 $\mathrm{Cp} \mathrm{p}^{*} \operatorname{Ir}(\mathrm{OAc})_{2}$ 作用可得到酰胺基导向的芳 基 $\mathrm{C}-\mathrm{H}$ 键活化关键中间体 37 , 其结构经单晶 $\mathrm{X}$ 射线衍 射证实. 37 可与 $\mathrm{Cu}(\mathrm{II})$ 氧化剂反应得到进一步 $\mathrm{B}-\mathrm{H} / \mathrm{N}-$ $\mathrm{H}$ 脱氢偶联产物 35, 控制实验和循环伏安实验结果证 实, 本步反应中存在 $\operatorname{Ir}(\mathrm{V})$-氮宾中间体, 通过 37 中的
$\mathrm{C}$ - Ir 键质子化和氮宾对 $\mathrm{B}-\mathrm{H}$ 键的迁移插入最终构筑 $\mathrm{B}-\mathrm{N}$ 键. 该工作对区域选择性和化学选择性可控的连 续脱氢偶联反应具有重要的参考价值.

羧基取代碳硼烷 34 同样也能与苯甲酸经过铱催化 $\mathrm{B}-\mathrm{H} / \mathrm{C}-\mathrm{H}$ 和 $\mathrm{B}-\mathrm{H} / \mathrm{O}-\mathrm{H}$ 的串联脱氢偶联反应, 高效 制备碳嗍烷并香豆素衍生物 38 (Scheme 17) ${ }^{[36]}$. 改变反 应条件同样可得到单一 $\mathrm{B}-\mathrm{H} / \mathrm{C}-\mathrm{H}$ 交叉偶联产物 39. 而与 Scheme 16 中苯甲酰胺反应不同的是，苯甲酸与碳 碍烷的第一步 $\mathrm{B}-\mathrm{H} / \mathrm{C}-\mathrm{H}$ 脱氢偶联完成后, 碳硼烷 $\mathrm{C}(1)$ 位的羧基导向基并不会发生原位脱除，而是进一步 导向后续的 $\mathrm{B}-\mathrm{H}$ 键活化，再经由氧化所得的 $\operatorname{Ir}(\mathrm{V})-\mathrm{B}$ 物 种与分子内苯甲酸羧基作用最终构建 $\mathrm{B}-\mathrm{O}$ 键. 该反应 中 $\mathrm{C}(1)$ 羧基导向基以及氧化剂的存在是连续脱氢偶联 的关键，羧基导向基在反应的最终步才进行脱除.

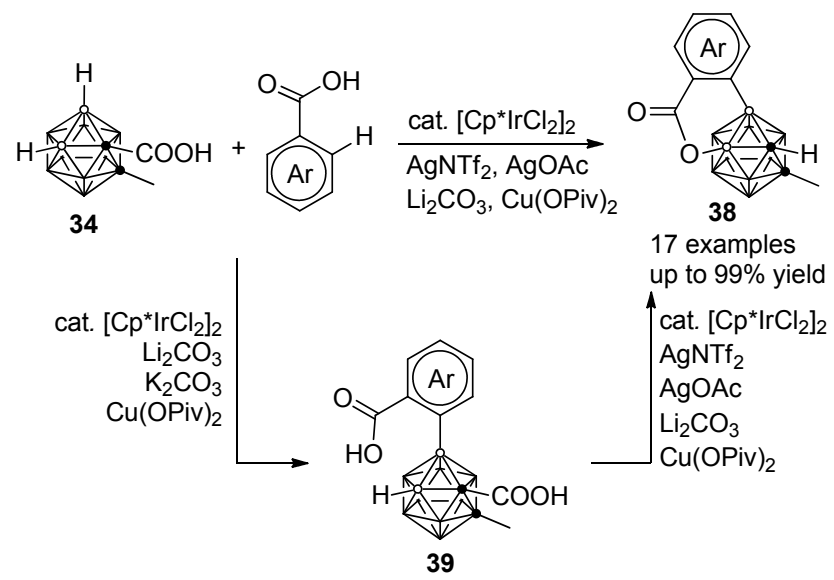

图式 17 铱催化 $\mathrm{BH} / \mathrm{CH}$ 和 $\mathrm{BH} / \mathrm{OH}$ 串联脱氢交叉偶联合成碳 嗍烷并香豆素

Scheme 17 Synthesis of carborano-coumarins via iridium catalyzed cascade dehydrogenative cross-coupling of $\mathrm{BH} / \mathrm{CH}$ and $\mathrm{BH} / \mathrm{OH}$ 
与 Scheme 12 中所示的一个碳顶点连有取代基的 3碘代一邻-碳硼烷 28 的反应不同, 炔烃可以直接插入 3碘代一邻-碳硼烷 40 与 $\mathrm{Pd}\left(\mathrm{PPh}_{3}\right)_{4}$ 催化剂作用所得的 $\mathrm{B}$ $\mathrm{Pd}$ 键, 而反应最终得到碘迁移至 B(4)位产物 41. DFT 理 论计算结果表明钯片段由 exo-烯基碳向 $\mathrm{B}(4)$ 顶点迁移的 过程 $(\mathbf{T} \rightarrow \mathbf{U})$ 在热力学上十分有利 $(\Delta G=-88.7 \mathrm{~kJ} / \mathrm{mol})$. 而基于已知的碘代一邻-碳硼烷钯催化 $\mathrm{B}-\mathrm{C}$ 偶联过 程 ${ }^{[37]}$, 利用上述烯基化-碘迁移串联反应中回收的 $\operatorname{Pd}(0)$ 催化剂进一步活化新生成 $\mathrm{B}(4)$ - $\mathrm{I}$ 键, 通过向体系中加 入不同的格氏试剂可以一锅法高效合成双官能团化邻碳硼烷 42 (Scheme 18). 结果表明, 一系列烷基/烯丙基/ 炔基/芳基格氏试剂都能应用于此反应, 并以优秀的收 率得到相应产物 ${ }^{[38]}$.

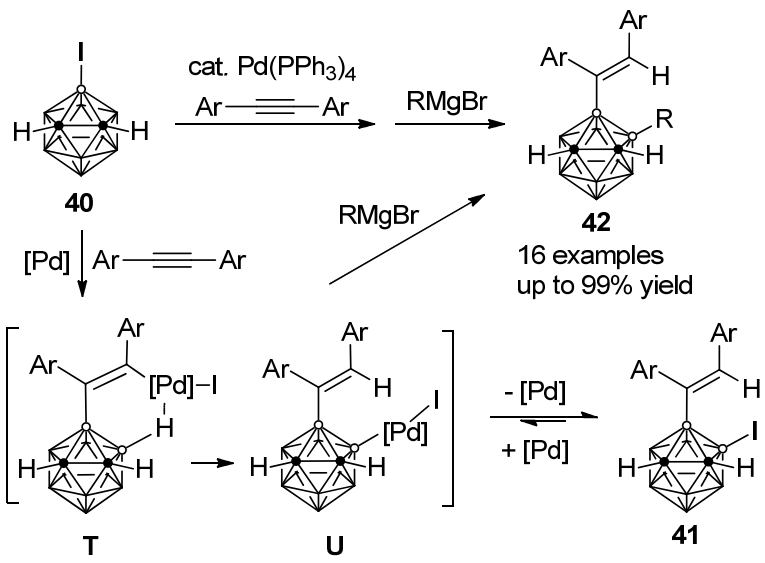

图式 18 钯迁移实现 3-碘代-邻-碳硼烷选择性一锅法双官能 团化

Scheme 18 Selective one-pot bifunctionalization of 3-iodo-ocarborane by Pd migration

与此同时, 在碘迁移步骤后采用不同的偶联试剂也 可以分别实现一锅法钯催化烯基化-氭基化/胺基化过 程, 以较高至优秀的收率得到 3-烯基-4-氧基/酰胺基邻一碳嗍烷 43 和 44 (Scheme 19) ${ }^{[38]}$. 氞代实验表明, B(4) 顶点的氛原子在反应后能以 $94 \%$ 的效率迁移到烯基上, 此结果为反应中的钯迁移关键步骤提供了确丵的证据. 该工作中高区域选择性双官能团化过程包含了对 B-I、 $\mathrm{B}(4)-\mathrm{H}$ 键的相继活化和由烯基碳至 B(4) 顶点钯迁移关 键步骤, 成功发展了一锅法在碳嗍烷硼顶点上引入两种 不同取代基的新路径.

\section{4 硼硼一取代十二硼烷阴离子衍生物的合成}

闭式十二硼烷阴离子 $\left[\mathrm{B}_{12} \mathrm{H}_{12}\right]^{2-}$ 同样具有二十面体 结构, 分子中十二个 $\mathrm{BH}$ 顶点完全等价, 是苯的三维类 似物. 它具有电子离域、高化学及热稳定性、低毒性的 特点, 在弱配位阴离子、配体、主-客体化学、催化、材 料及医药领域用作重要的结构单元 ${ }^{[39]}$. 通过各种官能

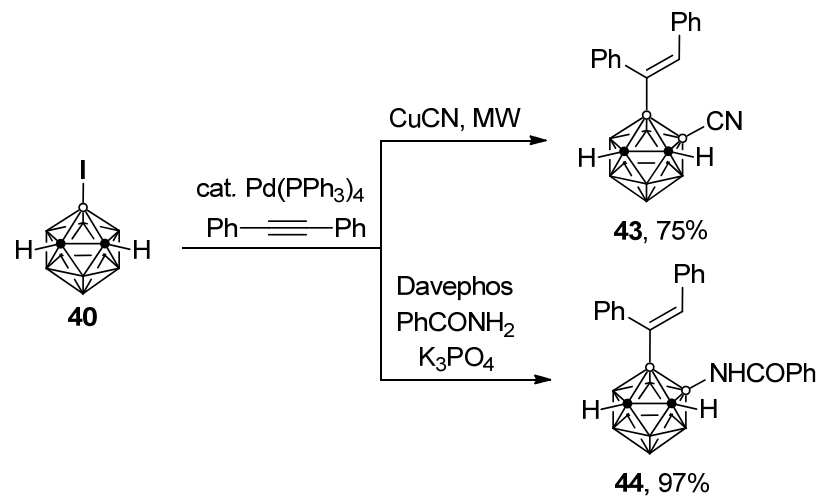

图式 19 钯催化一锅法烯基化-氰基化/酰胺基化过程 Scheme 19 Pd-catalyzed one-pot alkenylation-cyanation/amination

团对其进行修饰，可以为闭式十二硼烷的性质和应用研 究提供一个新的平台. 2016 年, Duttwyler 课题组 ${ }^{[40]}$ 发展 了过渡金属催化十二嗍烷阴离子区域选择性 $\mathrm{B}-\mathrm{H}$ 键活 化，实现复杂的 $1,2,3-$ 三取代 $(\mathrm{B}-\mathrm{C} / \mathrm{B}-\mathrm{O} / \mathrm{B}-\mathrm{N})$ 嗍烷衍 生物 46 的简单构筑(Scheme 20). 作者首先合成了含有 脲基导向基的十二硼烷阴离子 $\mathbf{4 5}$, 以 $\mathrm{Cu}(\mathrm{OAc})_{2} \bullet \mathrm{H}_{2} \mathrm{O}$ 为 氧化剂, 在 $\left[\mathrm{Cp} * \mathrm{RhCl}_{2}\right]_{2}$ 催化下与炔烃发生烯基化一分子 内环化串联反应，以最高 $85 \%$ 收率得到三取代的产物 46. 值得注意的是，此反应条件温和，在室温条件下即 具有良好的反应普适性以及较好的区域选择性.

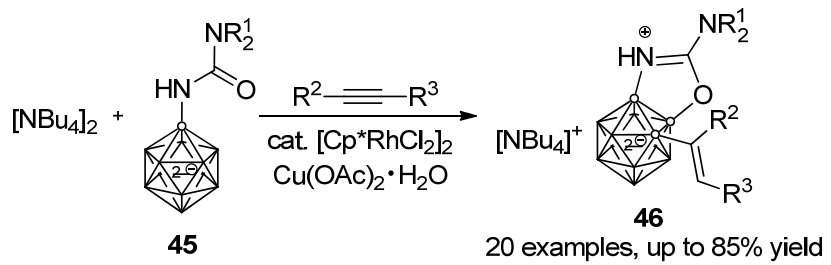

图式 20 铑催化闭式十二嗍烷阴离子经烯基化一环化串联反 应合成复杂三取代硼烷衍生物

Scheme 20 Synthesis of trisubstituted closo-dodecaborates by $\mathrm{Rh}$-catalyzed cascade alkenylation-annulation of closo-dodecaborate anions

化合物 45 与化学计量的 $\left[\mathrm{RhCp} *\left(\mathrm{CH}_{3} \mathrm{CN}\right)_{3}\right]\left[\mathrm{SbF}_{6}\right]_{2}$ 反应，可以高效生成中性含有抓氢键(agostic interaction) 的 $\mathrm{Rh}(\mathrm{III})$ 配合物 47 , 其结构经单晶 $\mathrm{X}$ 射线衍射证实. 进 一步通过控制实验对该反应的机理进行研究显示, 催化 循环中首先 $\left[\mathrm{Cp}^{*} \mathrm{RhCl}_{2}\right]_{2}$ 催化剂解离为更活跃的单体 $\mathrm{CpRh}{ }^{*} \mathrm{~L}_{2}\left(\mathrm{~L}=\right.$ 溶剂、 $\mathrm{Cl}^{-}$或 $\left.\mathrm{AcO}^{-}\right)$, 然后与嗍烷阴离子 45 络合形成中间体 47, 随后在 $\mathrm{AcO}^{-}$作用下发生 $\mathrm{B}-\mathrm{H}$ 键活化形成铑杂六元环中间体 $\mathbf{V}$, 反应物炔烃配位、插 入得到中间体 $\mathbf{W}$ ，随后质子化及金属 $\mathrm{Rh}$ 迁移形成中间 体 $\mathbf{X} . \mathrm{AcO}^{-}$可以进一步促进第二次 $\mathrm{B}-\mathrm{H}$ 键活化形成中 间体 $\mathbf{Y}$, 最后通过还原消除得到产物 $\mathbf{4 6}, \mathrm{Cu}(\mathrm{II})$ 可以将 $\mathrm{Rh}(\mathrm{I})$ 再次氧化为 $\mathrm{Rh}(\mathrm{III})$ 完成一次催化循环(Scheme 
$21)^{[40]}$

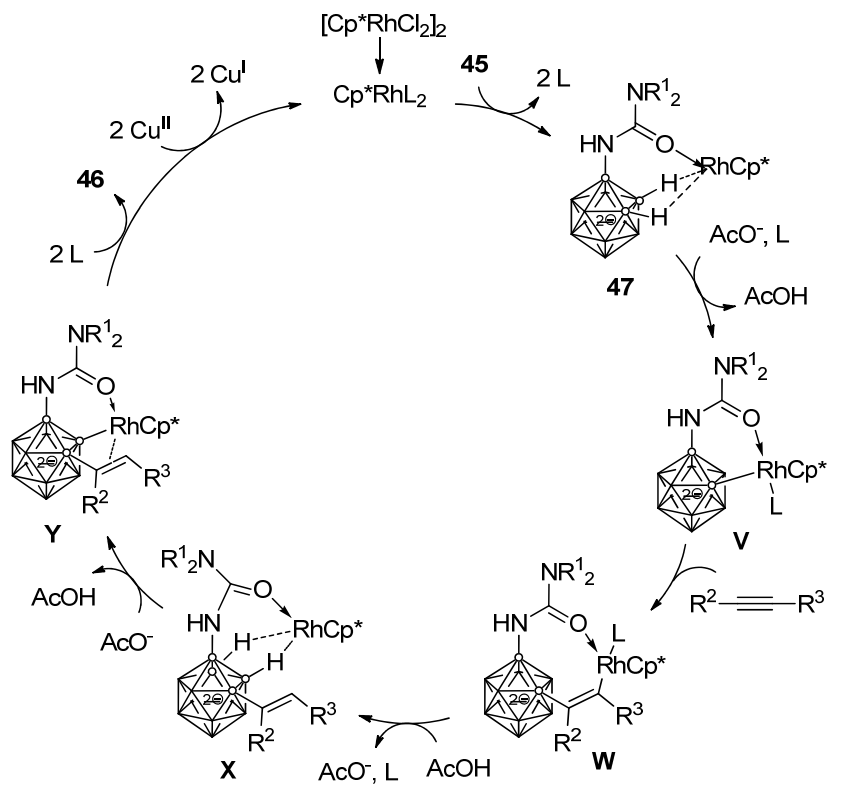

图式 21 铑催化双重 $\mathrm{B}-\mathrm{H}$ 键活化及烯基化-环化串联反应机 理

Scheme 21 Proposed mechanism for rhodium catalyzed cascade alkenylation-annulation through double $\mathrm{B}-\mathrm{H}$ activation

芳基酰胺作为导向基团同样可以促进闭式十二嗍 烷阴离子 48 与炔烃反应进行两个硼顶点的串联官能团 化反应, 该反应官能团耐受性广, 最高能以 $90 \%$ 的收率 得到三取代的硼烷衍生物 49.48 与芳基及烷基取代烯烃 反应，也可以得到类似的烷基化-环化产物 50 (Scheme $22)^{[41]}$. 值得注意的是, 反应中并未观察到导向基中芳 基 $\mathrm{C}-\mathrm{H}$ 键活化的产物, 证明本反应体系对 $\mathrm{B}-\mathrm{H}$ 和 $\mathrm{C}-\mathrm{H}$ 具有很好的化学选择性, 而反应的第二步环化过 程也具有完全的区域选择性. 所合成的复杂十二硼烷阴 离子衍生物将可以拓展嗍笼阴离子在发光材料和药物 化学领域应用研究.

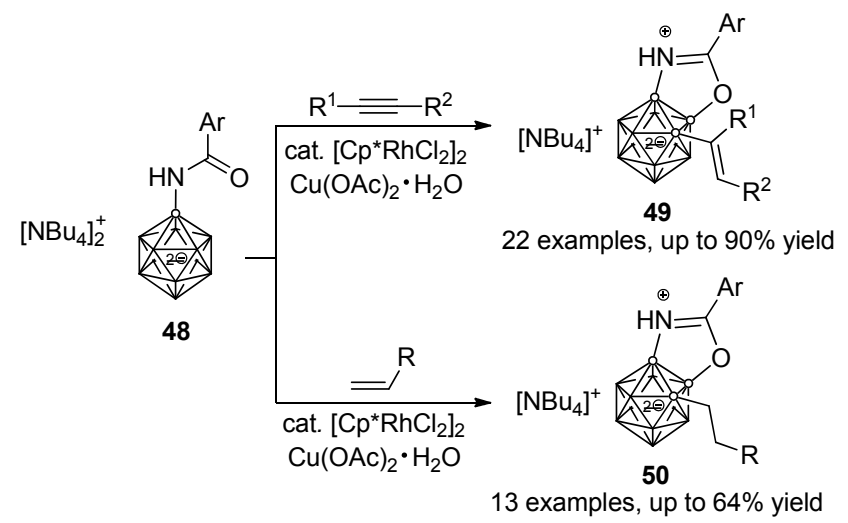

图式 22 铑催化酰胺基取代闭式十二硼烷阴离子烯基化/烷基 化-环化串联反应

Scheme 22 Rh-catalyzed cascade alkenylation/alkylation-annulation of closo-dodecaborate anions

\section{5 总结与展望}

本文综述了过渡金属促进的十二顶点碳硼烷与硼 烷阴离子的复杂官能团化过程，反应中涉及到多组分反 应、串联反应或一锅法反应的系列过程，具有高选择性、 操作步骤简单、高效实用的优点, 为复杂硼烷衍生物的 合成提供了新的方法和途径. 目前，过渡金属介导/催化 的硼烷官能团化反应是合成复杂硼烷衍生物的重要方 法之一，尽管近年来过渡金属催化的 $\mathrm{B}-\mathrm{H}$ 键活化研究 成果显著，但过渡金属催化的反应范围和机理仍有待进 一步探索. 另一方面, 这些高效 $\mathrm{B}-\mathrm{H}$ 键活化反应可以 很好地控制串联反应中的区域选择性，而如何在系列串 联反应中控制对映选择性而实现复杂手性硼烷化合物 的不对称合成是今后该领域的一个重要研究方向 ${ }^{[42]}$, 具有广阔的发展空间, 并能进一步开拓官能团化碳砋烷 及硼烷衍生物在医药、材料、催化等多个领域的应用前 景.

\section{References}

[1] (a) Grimes, R. N. Carboranes, 3rd ed., Academic Press, Amsterdam, 2016.

(b) Hosmane, N. S. Boron Science: New Technologies and Application, CRC Press, Boca Raton, FL, 2012

[2] (a) Scholz, M.; Hey-Hawkins, E. Chem. Rev. 2011, 111, 7035. (b) Leśnikowski, Z. J. J. Med. Chem. 2016, 59, 7738.

[3] (a) Hosmane, N. S.; Maguire, J. A. In Comprehensive Organometallic Chemistry III, Vol. 3, Eds.: Crabtree, R. H.; Mingos, D. M. P., Elsevier, Oxford, 2007, Chapter 5.

(b) Xie, Z. Acc. Chem. Res. 2003, 36, 1.

(c) Yao, Z.-J.; Jin, G.-X. Coord. Chem. Rev. 2013, 257, 2522.

(d) Qiu, Z.; Ren, S.; Xie, Z. Acc. Chem. Res. 2011, 44, 299.

(e) Estrada, J.; Lavallo, V. Angew. Chem. Int. Ed. 2017, 56, 9906

(f) El-Hellani, A.; Lavallo, V. Angew. Chem. Int. Ed. 2014, 53, 4489.

(g) Fisher, S. P.; Tomich, A. W.; Lovera, S. O.; Kleinsasser, J. F.; Guo, J.; Asay, M. J.; Nelson, H. M.; Lavallo, V. Chem. Rev. 2019, 119,8262 .

(h) Yao, Z.-J.; Yu, W.-B.; Lin, Y.-J.; Huang, S.-L.; Li, Z.-H.; Jin, G.-X. J. Am. Chem. Soc. 2014, 136, 2825.

(i) Gao, Y.; Guo, S.-T.; Cui, P.-F.; Aznarez, F.; Jin, G.-X. Chem. Commun. 2019, 55, 210.

(j) Cui, P.-F.; Gao, Y.; Guo, S.-T.; Lin, Y.-J.; Li, Z.-H.; Jin, G.-X. Angew. Chem. Int. Ed. 2019, 58, 8129.

[4] (a) Jude, H.; Disteldorf, H.; Fischer, S.; Wedge, T.; Hawkridge, A. M.; Arif, A. M.; Hawthorne, M. F.; Muddiman, D. C.; Stang, P. J. J. Am. Chem. Soc. 2005, 127, 12131.

(b) Koshino, M.; Tanaka, T.; Solin, N.; Suenaga, K.; Isobe, H.; Nakamura, E. Science 2007, 316, 853.

(c) Dash, B. P.; Satapathy, R.; Gaillard, E. R.; Maguire, J. A.; Hosmane, N. S. J. Am. Chem. Soc. 2010, 132, 6578

(d) Kung, C.-W.; Otake, K.; Buru, C. T.; Goswami, S.; Cui, Y; Hupp, J. T.; Spokoyny, A. M.; Farha, O. K. J. Am. Chem. Soc. 2018, 140, 3871.

(e) Villagómez, C. J.; Sasaki, T.; Tour, J. M.; Grill, L. J. Am. Chem. Soc. 2010, 132, 16848.

(f) Qian, E. Q.; Wixtrom, A. I.; Axtell, J. C.; Saebi, A.; Rehak, P.; Han, Y.; Moully, E. H.; Mosallaei, D.; Chow, S.; Messina, M.; Wang, J.-Y.; Royappa, A. T.; Rheingold, A. L.; Maynard, H. D.; Kral, P.; Spokoyny, A. M. Nat. Chem. 2017, 9, 333.

(g) Saha, A.; Oleshkevich, E.; Viñas, C.; Teixidor, F. Adv. Mater. 
2017, 29, 1704238.

(h) Guo, J.; Liu, D.; Zhang, J.; Zhang, J.; Miao, Q.; Xie, Z. Chem. Commun. 2015, 51, 12004.

(i) Jung, D.; Saleh, L. M. A.; Berkson, Z. J.; El-Kady, M. F.; Hwang, J. Y.; Mohamed, N.; Wixtrom, A. I.; Titarenko, E.; Shao, Y.; McCarthy, K.; Guo, J.; Martini, I. B.; Kraemer, S.; Wegener, E. C.; Saint-Cricq, P.; Ruehle, B.; Langeslay, R. R.; Delferro, M.; Brosmer, J. L.; Hendon, C. H.; Gallagher-Jones, M.; Rodriguez, J.; Chapman, K. W.; Miller, J. T.; Duan, X.; Kaner, R. B.; Zink, J. I.; Chmelka, B. F.; Spokoyny, A. M. Nat. Mater. 2018, 17, 341.

(j) Cui, P.-F.; Lin, Y.-J.; Li, Z.-H.; Jin, G.-X. J. Am. Chem. Soc. 2020, 142, 8532 .

[5] (a) Mukherjee, S.; Thilagar, P. Chem. Commun. 2016, 52, 1070.

(b) Núñez, R.; Tarrés, M.; Ferrer-Ugalde, A.; de Biani, F. F.; Teixidor, F. Chem. Rev. 2016, 116, 14307.

(c) Li, X.; Yan, H.; Zhao, Q. Chem. Eur. J. 2016, 22, 1888.

[6] (a) Heying, T. L.; Ager, J. W.; Clark, S. L.; Mangold, D. J.; Goldstein, H. L.; Hillman, M.; Polak, R. J.; Szymanski, J. W. Inorg. Chem. 1963, 2, 1089.

(b) Fein, M. M.; Bobinski, J.; Mayes, N.; Schwartz, N.; Cohen, M. S. Inorg. Chem. 1963, 2, 1111.

(c) El-Zaria, M. E., Keskar, K., Genady, A. R., Ioppolo, J. A., McNulty, J.; Valliant, J. F. Angew. Chem. Int. Ed. 2014, 53, 5156.

[7] (a) Wu, S.; Jones, M., Jr. Inorg. Chem. 1986, 25, 4802.

(b) Bregadze, V. I. Chem. Rev. 1992, 92, 209.

(c) Viñas, C.; Benakki, R.; Teixidor, F.; Casabó, J. Inorg. Chem. 1995, 34, 3844 .

(d) Gomez, F. A.; Johnson, S. E.; Hawthorne, M. F. J. Am. Chem. Soc. 1991, 111, 5915.

(e) Gomez, F. A.; Hawthorne, M. F. J. Org. Chem. 1992, 57, 1384. (f) Anderson, K. P.; Mills, H. A.; Mao, C.; Kirlikovali, K. O.; Axtell, J. C.; Rheingold, A. L.; Spokoyny, A. M. Tetrahedron 2019, 75, 187.

[8] (a) Lu, J.-Y.; Wan, H.; Zhang, J.; Wang, Z.; Li, Y.; Du, Y.; Li, C.; Liu, Z.-T.; Liu, Z.-W.; Lu, J. Chem.-Eur. J. 2016, 22, 17542.

(b) Tang, C.; Xie, Z. Angew. Chem. Int. Ed. 2015, 54, 7662.

(c) Xie, Z. Sci. China Chem. 2014, 57, 1061

(d) Coult, R.; Fox, M. A.; Gill, W. R.; Herbertson, P. L.; MacBride, J. A. H.; Wade, K. J. Organomet. Chem. 1993, 462, 19.

[9] (a) Tang, C.; Zhang, J.; Xie, Z. Angew. Chem. Int. Ed. 2017, 56, 8642 .

(b) Tang, C.; Zhang, J.; Zhang, J.; Xie, Z. J. Am. Chem. Soc. 2018, 140, 16423.

[10] Lyu, H.; Zhang, J.; Yang, J.; Quan, Y.; Xie, Z. J. Am. Chem. Soc. 2019, 141, 4219.

(b) Quan, Y.; Xie, Z. J. Am. Chem. Soc. 2015, 137, 3502.

(c) Quan, Y.; Xie, Z. Angew. Chem. Int. Ed. 2016, 55, 1295.

(d) Zhang, X.; Yan, H. Chem. Sci. 2018, 9, 3964.

(e) Zhang, X.; Zheng, H.; Li, J.; Xu, F.; Zhao, J.; Yan, H. J. Am. Chem. Soc. 2017, 139, 14511.

(f) Cao, K.; Huang, Y.; Yang, J.; Wu, J. Chem. Commun. 2015, 51, 7257.

(g) Lin, F.; Yu, J.-L.; Shen, Y.; Zhang, S.-Q.; Spingler, B.; Liu, J.; Hong, X.; Duttwyler, S. J. Am. Chem. Soc. 2018, 140, 13798.

(h) Xu, T. T.; Cao, K.; Zhang, C. Y.; Wu, J.; Ding, L. F.; Yang, J. Org. Lett. 2019, 21, 9276.

(i) Xu, T. T.; Cao, K.; Zhang, C. Y.; Wu, J.; Jiang, L.; Yang, J. Chem. Commun. 2018, 54, 13603.

[11] (a) Liang, X.; Shen, Y.; Zhang, K.; Liu, J.; Duttwyler, S. Chem. Commun. 2018, 54, 12451.

(b) Lin, F.; Shen, Y.; Zhang, Y.; Sun, Y.; Liu, J.; Duttwyler, S. Chem. Eur. J. 2018, 24, 551.

(c) Lyu, H.; Quan, Y.; Xie, Z. Angew. Chem. Int. Ed. 2015, 54, 10623.

(d) Quan, Y.; Xie, Z. J. Am. Chem. Soc. 2014, 136, 15513.

(e) Mirabelli, M. G. L.; Sneddon, L. G. J. Am. Chem. Soc. 1988, 110,449 .

(f) Wu, J.; Cao, K.; Xu, T.-T.; Zhang, X.-J.; Jiang, L.; Yang, J.;
Huang, Y., RSC Adv. 2015, 5, 91683.

(g) Shen, Y.; Pan, Y.; Zhang, K.; Liang, X.; Liu, J.; Spingler, B.; Duttwyler, S. Dalton Trans. 2017, 46, 3135

(h) Shen, Y.; Zhang, K.; Liang, X.; Dontha, R.; Duttwyler, S. Chem. Sci. 2019, 10, 4177.

(i) Cheng, R.; Qiu, Z.; Xie, Z. Chem. Eur. J. 2020, 26, 7121

[12] (a) Chen, Y.; Au, Y. K.; Quan, Y.; Xie, Z. Sci. China Chem. 2019, $62,74$.

(b) Quan, Y.; Tang, C.; Xie, Z. Chem. Sci. 2016, 7, 5838.

[13] Cheng, R.; Qiu, Z.; Xie, Z. Nat. Commun. 2017, 8, 14827.

[14] Qiu, Z.; Quan, Y.; Xie, Z. J. Am. Chem. Soc. 2013, 135, 12192.

[15] (a) Cui, C. X.; Zhang, J.; Qiu, Z.; Xie, Z. Dalton Trans. 2020, 49, 1380 .

(b) Au, Y. K.; Lyu, H.; Quan, Y.; Xie, Z. J. Am. Chem. Soc. 2020 142, 6940 .

(c) Lyu, H.; Quan, Y.; Xie, Z. Angew. Chem. Int. Ed. 2016, 55, 11840

(d) Cao, K.; Xu, T.-T.; Wu, J.; Jiang, L.; Yang, J. Chem. Commun. 2016, $52,11446$.

(e) Li, C.-X.; Zhang, H.-Y.; Wong, T.-Y.; Cao, H.-J.; Yan, H.; Lu, C.-S. Org. Lett. 2017, 19, 5178.

[16] Lyu, H.; Quan, Y.; Xie, Z. J. Am. Chem. Soc. 2016, 138, 12727.

[17] Lyu, H.; Quan, Y.; Xie, Z. Chem. Eur. J. 2017, 23, 14866.

[18] (a) Quan, Y.; Qiu, Z.; Xie, Z. Chem. Eur. J. 2018, 24, 2795.

(b) Quan, Y.; Xie, Z. Chem. Soc. Rev. 2019, 48, 3660.

(c) Yu, W.-B.; Cui, P.-F.; Gao, W.-X.; Jin, G.-X. Coord. Chem. Rev. 2017, 350, 300.

(d) Zhang, X.; Yan, H. Coord. Chem. Rev. 2019, 378, 466.

[19] Qiu, Z.; Ren, S.; Xie, Z. Acc. Chem. Res. 2011, 44, 299.

[20] Qiu, Z.; Xie, Z. J. Am. Chem. Soc. 2009, 131, 2084.

[21] Deng, L.; Chan, H.-S.; Xie, Z. J. Am. Chem. Soc. 2006, 128, 7728

[22] Ren, S.; Chan, H.-S.; Xie, Z. Organometallics 2009, 28, 4106.

[23] Ren, S.; Qiu, Z.; Xie, Z. J. Am. Chem. Soc. 2012, 134, 3242.

[24] Ren, S.; Chan, H.-S.; Xie, Z. J. Am. Chem. Soc. 2009, 131, 3862.

[25] Ren, S.; Qiu, Z.; Xie, Z. Organometallics 2012, 31, 4435.

[26] Ren, S.; Qiu, Z.; Xie, Z. Angew. Chem. Int. Ed. 2012, 51, 1010.

[27] Quan, Y.; Zhang, J.; Xie, Z. J. Am. Chem. Soc. 2013, 135, 18742.

[28] Zhang, J.; Quan, Y.; Lin, Z.; Xie, Z. Organometallics 2014, 33, 3556.

[29] Cui, C.-X.; Ren, S.; Qiu, Z.; Xie, Z. Dalton Trans. 2018, 47, 2453.

[30] Quan, Y.; Qiu, Z.; Xie, Z. J. Am. Chem. Soc. 2014, 136, 7599.

[31] Wang, Z.; Ye, H.; Li, Y.; Li, Y.; Yan, H. J. Am. Chem. Soc. 2013, $135,11289$.

[32] Zhang, R.; Zhu, L.; Liu, G.; Dai, H.; Lu, Z.; Zhao, J.; Yan, H. J. Am. Chem. Soc. 2012, 134, 10341.

[33] Qiu, Z.; Xie, Z. J. Am. Chem. Soc. 2010, 132, 16085.

[34] Lyu, H. Quan, Y.; Xie, Z. Chem. Sci. 2018, 9, 6390.

[35] Au, Y.-K.; Lyu, H.; Quan, Y.; Xie, Z. J. Am. Chem. Soc. 2019, 141, 12855.

[36] Au, Y.-K.; Lyu, H.; Quan, Y.; Xie, Z. Chin. J. Chem. 2020, 38, 383.

[37] (a) Li, J.; Logan, C. F.; Jones, M. Jr. Inorg. Chem. 1991, 30, 4866.

(b) Zheng, Z.; Jiang, W.; Zinn, A. A.; Knobler, C. B.; Hawthorne, M. F. Inorg. Chem. 1995, 34, 2095.

(c) Harakas, G.; Vu, T.; Knobler, C. B.; Hawthorne, M. F. J. Am. Chem. Soc. 1998, 120, 6405

(d) Viñas, C.; Barberà, G.; Oliva, J. M.; Teixidor, F.; Welch, A. J.; Rosair, G. M. Inorg. Chem. 2001, 40, 6555.

[38] Ge, Y.; Zhang, J.; Qiu, Z.; Xie, Z. Angew. Chem. Int. Ed. 2020, 59, 4851.

[39] (a) Poater, J.; Solà, M.; Viñas, C.; Teixidor, F. Chem. Eur. J. 2016, $22,7437$.

(b) Axtell, J. C.; Saleh, L. M. A.; Qian, E. A.; Wixtrom, A. I.; Spokoyny, A. M. Inorg. Chem. 2018, 57, 2333.

(c) Bolli, C.; Derendorf, J.; Keßler, M.; Knapp, C.; Scherer, H.; Schulz, C.; Warneke, J. Angew. Chem. Int. Ed. 2010, 49, 3536.

(d) Kirchmann, M.; Wesemann, L. Dalton Trans. 2008, 2144.

(e) Messina, M. S.; Axtell, J. C.; Wang, Y.; Chong, P.; Wixtrom, A. I.; Kirlikovali, K. O.; Upton, B. M.; Hunter, B. M.; Shafaat, O. S.; 


\section{REVIEW}

Khan, S. I.; Winkler, J. R.; Gray, H. B.; Alexandrova, A. N.; Maynard, H. D.; Spokoyny, A. M. J. Am. Chem. Soc. 2016, 138, 6952.

(f) Peryshkov, D. V.; Strauss, S. H. Inorg. Chem. 2017, 56, 4072. (g) Wang, W.; Wang, X.; Cao, J.; Liu, J.; Qi, B.; Zhou, X.; Zhang, S.; Gabel, D.; Nau, W. M.; Assaf, K. I.; Zhang, H. Chem. Commun. 2018, 54, 2098.

(h) Assaf, K. I.; Ural, M. S.; Pan, F.; Georgiev, T.; Simova, S.; Rissanen, K.; Gabel, D.; Nau, W. M. Angew. Chem. Int. Ed. 2015, 54, 6852.

(i) Assaf, K. I.; Hennig, A.; Peng, S.; Guo, D.-S.; Gabel, D.; Nau, W. M. Chem. Commun. 2017, 53, 4616.

(j) Jung, D.; Saleh, L. M. A.; Berkson, Z. J.; El-Kady, M. F.;
Hwang, J. Y.; Mohamed, N.; Wixtrom, A. I.; Titarenko, E.; Shao, Y.; McCarthy, K.; Guo, J.; Martini, I. B.; Kraemer, S.; Wegener, E. C.; Saint-Cricq, P.; Ruehle, B.; Langeslay, R. R.; Delferro, M.; Brosmer, J. L.; Hendon, C. H.; Gallagher-Jones, M.; Rodriguez, J.; Chapman, K. W.; Miller, J. T.; Duan, X.; Kaner, R. B.; Zink, J. I.; Chmelka, B. F.; Spokoyny, A. M. Nat. Mater. 2018, 17, 341.

[40] Zhang, Y.; Sun, Y.; Lin, F.; Liu, J.; Duttwyler, S. Angew. Chem. Int. Ed. 2016, 55, 15609.

[41] Zhang, Y.; Wang, T.; Wang, L.; Sun, Y.; Lin, F.; Liu, J.; Duttwyler, S. Chem. Eur. J. 2018, 24, 15812.

[42] Cheng, R.; Li, B.; Wu, J.; Zhang, J.; Qiu, Z.; Tang, W.; You, S. L.; Tang, Y.; Xie, Z. J. Am. Chem. Soc. 2018, 140, 4508.

(Fan, Y.) 